\title{
Deadline Scheduling for Large Scale Charging of Electric Vehicles with Renewable Energy
}

\author{
Shiyao Chen, Yuting Ji, and Lang Tong \\ School of Electrical and Computer Engineering \\ Cornell University, Ithaca, NY 14850 \\ $\{$ sc933,yj246, lt35\}@cornell.edu
}

\begin{abstract}
The problem of scheduling for the large scale charging of electric vehicles with renewable sources is considered. A new online charging algorithm referred to as Threshold Admission with Greedy Scheduling (TAGS) is proposed by formulating the charging problem as one of deadline scheduling with admission control and variable charging capacities. TAGS has low computation cost and requires no prior knowledge on the distributions of arrival traffic, battery charging (service) time, and available energy from renewable sources. It has a reserve dispatch algorithm designed to compensate the intermittency of renewable sources. Performance of TAGS is compared with benchmark scheduling algorithms such as the Earliest Deadline First (EDF) and the First Come First Serve (FCFS) with aggressive and conservative reserve dispatch algorithms.

Index Terms-EV/PHEV charging, smart grid, demand response, renewable energy, deadline scheduling.
\end{abstract}

\section{INTRODUCTION}

We consider the problem of scheduling the charging of a large number of vehicles with intermittent energy sources. This problem is motivated by the need for developing infrastructures at public parking facilities, work places, and apartment complexes where a large number of EVs can be charged simultaneously. Such a Large Scale Charging (LSC) infrastructure is essential for densely populated urban areas, for which electrified transportation system is essential but inhome EV charging is not an option. LSC can also be a demand response mechanism that viewing $\mathrm{EV}$ charging as managing a large number of deferrable loads based on the availability of renewable resources and energy supply from the grid. To maximize its demand response power and potential, an LSC operator can provide incentives to the customers for offering flexible charging schedules and exploit such flexibilities. With a large number of in-facility EVs, the LSC operator also has the potential to offer ancillary and regulation services to the grid.

Figure 1 illustrates an architecture of the LSC operation considered in this paper. We focus here on the design of LSC Energy Management System (EMS) with the goal of maximizing the profit of its operator. The EMS controls a charging switching network that activates or de-activates the charging of in-facility EVs. To exploit renewable sources that are intermittent and unpredictable, the EMS jointly schedules

This research was sponsored in part by the National Science Foundation under a Grant CNS-1135844.

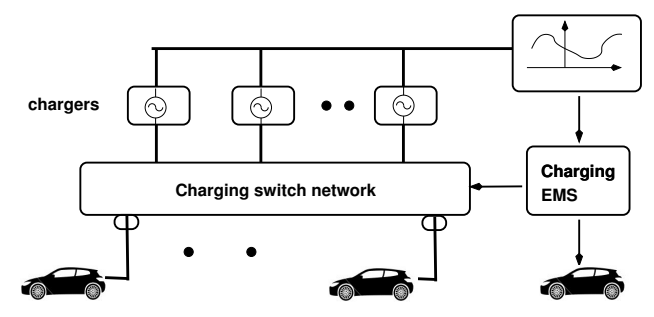

Figure 1. An architecture for large scale EV charging.

reserve dispatch and $\mathrm{EV}$ charging. The reserve can be in the form of collocated fast generation units, and it can be tapped for generation when the renewable energy is insufficient to sustain the LSC operation. To this end, we propose a profit based reserve dispatch scheme coupled with a deadline scheduling algorithm that exploits the variability in renewable energy supply, the reserve capacity, and the customer's flexible schedule.

We adopt the framework of online scheduling of charging requests where it is assumed that a customer arriving at a charging facility can communicate her charging needs (the amount of charging required and the deadline for completion) to the operator. Without reservation requirement, the operator has to decide upon customer arrival, whether to accept the customer request.

A key feature of the proposed scheduling algorithm is its admission control. It is almost inevitable that an online deadline scheduling algorithm operating in an uncertain environment cannot complete all the accepted charging requests. In the context of LSC using renewable sources, temporary shortages in supply may affect the completion of accepted charging requests. In such cases, unsatisfied customers have to be compensated. Thus the admission control plays an important role in reducing the costs due to incompletion.

Beside maximizing profit, managing LSC requires a computationally scalable scheduling algorithm with low implementation complexity. Because the performance of any algorithm depends on the characteristics of EV arrivals, their charging needs, and the availability of power from renewable sources, designing the optimal scheduling algorithm is in general intractable even if the joint distribution of uncertainties is known. We adopt a deterministic approach by working directly on the 
sample path and propose a simple online admission/scheduling algorithm. Referred to as Threshold Admission with Greedy Scheduling (TAGS), the proposed algorithm uses a simple threshold test for admission and a greedy algorithm for scheduling.

\section{A. Related Work}

A variety of modeling and optimization techniques have been proposed for EV charging scheduling problem. The authors of [1] propose an offline decentralized protocol for negotiating day-ahead charging prices and schedules for household EV charging between the EV owners and utility, to shift the charging load to fill the overnight demand valley. The household EV charging scheduling problem is casted into an optimal power flow (OPF) problem in [2] and the solution structure of $\mathrm{OPF}$ is leveraged for charging scheduling. The EV charging for public garages (with firm energy sources) has been considered in [3] with a heuristic optimization approach. A recent work [4] by Subramanian et. al. is one of the earliest addressing the use of renewable energy for EV charging and the need of considering reserves. Without admission control, the proposed algorithms aim to satisfy all requests with the help of reserve energy. In [5], [6] the authors consider the management of EV charging with the potential speculation in the provision of additional regulation service required by renewable energy expansion and propose a rolling horizon look-ahead stochastic dynamic programming. The authors of [7] propose the idea of using parking facility as an energy exchange station called "smart garage" for Vehicle-to-Grid (V2G) applications. They demonstrate the benefits of using EVs as energy storage for demand side management. In [8] a decentralized algorithm is proposed to coordinate the autonomous EV charging in non-cooperative game framework, which converges to a Nash equilibrium that approximately achieves the ideal solution (scheduling EV load to fill the overnight demand valley).

The proposed algorithm TAGS is an extension of an earlier approach first proposed in [9], [10]. The extension is made in several directions, most significant is to handle variable sources of power supply by casting the problem as one of multi-processor deadline scheduling. While TAGS has its root in the classical deadline scheduling problems, it should be noted that the traditional deadline scheduling formulation does not include non-completion penalty. Since the seminal work of Liu and Layland [11], there is considerable literature addressing the scheduling problem in the underloaded and overloaded regimes. For the underloaded scenario with single processor, simple online algorithms such as Earliest Deadline First (EDF) [11] and Least Laxity First (LLF) [12] achieve the same performance as the optimal offline algorithm. When the restrictive and unverifiable assumption of underloaded workload is relaxed, Locke showed in [13] that both EDF and LLF can perform poorly in the presence of overload. There were efforts to develop an online scheduling algorithm with worst case performance guarantee when the system is overloaded in [14].

\section{EV CHARGING MODEL}

We assume constant power charging for all the requests (with power $m$ ). We encapsulate the instantaneous charging capability of renewable energy sources by the notion of number of renewable chargers at time $t, n(t)=\lfloor w(t) / m\rfloor$ where $w(t)$ is the available renewable power supply at time $t$. Each EV charging request $T=(r, p, d)$ is represented by a triple specified by the arrival (release) time $r$, requested charging (processing) time $p$, and deadline $d$. Each fulfilled customer request is associated with revenue equal to the price of the specific request $v(r, d, p)$, which is a function of the request parameters $r, d$ and $p$, whereas each admitted but unfulfilled request is associated with a non-completion penalty $\frac{\hat{p}}{p} v(r, d, p)$, where $\hat{p}$ is the unfulfilled amount of the requested charging level and the non-completion penalty is proportional to the unfinished percentage $\frac{\hat{p}}{p}$. This specific non-completion penalty suits LSC well since utility is delivered to the EV owner continuously as the battery level increases.

The price function $v(r, d, p)$ should be tied to both the requested charging level and the tightness of the deadline, since this provides an incentive for the customers to consider their flexibility and submit charging requests with relaxed deadlines whenever possible. Specifically, we adopt the price function $v(r, d, p)=p h\left(\frac{d-r}{p}\right)$ in the simulations in Section IV, where $h$ is a decreasing function of the relative deadline ratio $\frac{d-r}{p}$, indicating the incentive given to relaxed deadlines. Additional reserve chargers that draw energy from the power grid or fast generation units are available at the cost of $c$ per unit time per charger, i.e., a request with processing time $p$ could be fulfilled by a reserve charger at cost $c p$. In LSC operations, preemption is allowed at no cost, i.e., a preempted battery can be resumed charging from the previous battery level upon preemption.

Upon receiving a customer request the LSC operator can decline the request, since, e.g., the facility is currently busy serving more profitable requests; or accept the request subject to the non-completion liability or reserve energy bill. The profit for the LSC operator is the revenue of all completed charging requests, less penalties paid for the admitted requests that miss their deadlines, less the reserve energy cost incurred. The reserve dispatch, admission and scheduling have to be conducted in an online fashion by the LSC operator aiming at satisfactory profit performance, i.e., the LSC operator is not aware of the future requests when making decisions.

\section{RESERVE Dispatch, Admission, AND SCHEDUling}

Intuitively, the admission and scheduling tend to be easy for the LSC operator if the overall charging load from the customer requests is well below the level of the renewable energy supply. Indeed, when the overall charging load is low, traditional algorithms such as EDF and First Come First Serve (FCFS) show reasonable performance by simply admitting all requests that ever arrive without incurring much reserve energy cost. However, if overwhelmingly many charging requests arrive in a short period of time, e.g., during rush hours and large public events, or days when renewable energy supply is 
low, the admission and scheduling will be more challenging, and the dispatch of reserve energy has to be taken into consideration.

The LSC operator maintains a tentative schedule for each currently available renewable charger and makes reserve dispatch and admission/scheduling decisions upon the following events: 1) new request releases (admission/scheduling decision and/or reserve dispatch decision), 2) a renewable charger becoming available/unavailable (request redistribution decision and/or reserve dispatch decision upon instantaneous renewable power supply increases/decreases).

\section{A. TAGS: Admission and Scheduling}

When a customer request arrives and finds the facility running well below renewable energy supply capacity, the LSC operator immediately admits the customer, and assigns the request to one of the lightly loaded chargers in an EDF manner. When a customer request arrives to a heavily occupied facility and cannot be accommodated in an EDF fashion, the LSC operator faces a dilemma that admitting the customer may lead to non-completion liability, while declining the customer means losing profit at hand. While optimal tradeoff between the two concerns is difficult and involves accommodating the newly arrived customer with minimum non-completion liability, we propose a threshold admission with greedy scheduling approach.

The key idea is to evaluate the admission decision based on the comparison of the potential profit associated with admitting and declining the customer request. Specifically, the LSC operator enumerates the potential renewable chargers. For each renewable charger the admitting option is evaluated by considering the price associated with the released request as well as the potential reserve energy cost and non-completion penalty incurred; the declining option is evaluated by recognizing the potential revenue of the requests that would have been affected upon admitting the new request. The previous requests may be affected and failed, or affected and forced to resort to the reserve energy. The ratio of the profit associated with admitting and declining is computed for each potential renewable charger. Only if the maximum ratio is over a prescribe threshold, the operator will admit this request and assign it to the renewable charger with the maximum ratio.

TAGS evaluates the potential profit of accepting to a renewable charger with one alternative of scheduling decision for the newly released request $T=(r, p, d)$. Specifically, when evaluating the option of accepting to charger $k$, the alternative of scheduling $T$ proceeds as follows. The tentative schedule of charger $k$ is updated by tight-scheduling $T$ in the interval $[d-p, d]$. Then the part of the previous tentative schedule after time $d-p$ is moved to start at time $d$, or the end of the current schedule, whichever comes later in time. This movement may lead to some of the moved jobs missing their deadlines, and the tentative schedule is updated by removing the part of the moved jobs that comes after their deadlines.

After admitting the request and assigning the charger, TAGS makes the scheduling decision in a greedy manner. Specifi- cally, if the operator decides to admit the request and assign to charger $k$, the tentative schedule of charger $k$ is updated by EDF-scheduling the previous requests in the tentative schedule as well as the newly released request. As we have mentioned, the newly released request cannot be accommodated in an EDF fashion. Therefore some requests in the previous tentative schedule may miss their deadlines. The decision of whether to dispatch reserve for the missed processing is made by weighing the reserve cost and the non-completion penalty. The ultimate scheduling decision follows EDF fashion since EDF performs well in lightly loaded regime, and TAGS ensures that the set of requests accepted into the facility is within facility capacity.

To summarize, when the LSC operator decides to admit the newly released request, the request is profitable once accepted but difficult to accommodate into the current schedule. Therefore the operator sacrifices some jobs in the current tentative schedule, some of which may have deadlines far into the future, thus still have potential in completion, while others will either resort to reserve energy, or be left unfinished depending on the incurred cost.

\section{B. Reserve Energy Dispatch and Request Redistribution}

With the reserve energy at disposal the LSC operator has to decide whether the profit from the fulfilled requests is worth the electricity bill incurred. The reserve energy may be dispatched when there is excessive arrival of profitable requests, and when the renewable power availability goes down in time, leaving the already admitted requests risky.

The redistribution of already admitted requests is due to the variability of the renewable energy source over time. Specifically, when a renewable charger becomes available, the LSC operator redistributes the requests that were previously arranged to complete with reserve energy to the newly available renewable charger to cut the reserve energy bill and non-completion liability. The LSC operator collects the requests that are still within their deadlines and were previously expected to fail or incur reserve energy supply. All collected requests go through the admission/scheduling of TAGS algorithm with the additional renewable charger. Similarly, when one renewable charger becomes unavailable, the requests currently in its tentative schedule are at risk. The LSC operator redistributes the risky requests to the remaining renewable chargers, and the LSC operator may find it necessary to incur additional reserve chargers.

When a newly released request gets rejected by TAGS, the LSC operator decides not to spend renewable energy on the request. However, the request may still be fulfilled with reserve energy if profitable. The request price and the reserve energy cost are compared to decide the profitability to incur additional reserve charger for the newly released request.

\section{Average Performance: Simulation Results}

We simulate the average performance of the profit based reserve dispatch with TAGS algorithm and compare with EDF and FCFS with a simple admission control scheme and 
conservative/aggressive reserve dispatch. The admission control scheme of EDF and FCFS is implemented for conservative(aggressive) reserve dispatch as follows: the LSC operator declines(resorts to reserve energy for) a newly released request if overload is detected for all available renewable chargers once the request is incorporated into the tentative schedule. That is, the facility operator maintains the feasibility of the requests accepted so far for renewable chargers. Conservative reserve dispatch always declines a request if feasibility cannot be maintained, while aggressive reserve dispatch always resorts to reserve energy. In Fig. 2 the performance of EDF and FCFS is normalized by that of TAGS, and the percentage is plotted versus the arrival rate.

As for the request parameters we assume the customer arrival process Poisson with parameter $\lambda$, the maximum requested charging time 120 (full battery charging), the requested battery charging percentage $X$ distributed in the support $[0,1]$ with pdf $2 x$ (i.e., requests tend to have long charging duration), and the relative deadlines exponentially distributed with mean 150 , where the relative deadline of request $T=(r, d, p)$ is $d-r-p$. The price function is set to be $v(r, d, p)=p\left(1+1_{\{(d-r) / p<2\}}\right)$. The reserve energy cost coefficient $c=1.9$. The threshold of TAGS is taken to be 3 . We conduct 30 Monte Carlo runs with 10000 jobs released over time for each Monte Carlo run. The renewable power supply trajectory is taken from the wind power generation spreadsheet provided by Bonneville Power Administration with duration of 24 hours.

We simulate a series of arrival rate $\lambda$ that covers both underloaded (left hand side of $x$ axis) and overloaded (right hand side of $x$ axis) scenarios, and compare EDF/FCFS with TAGS. It can be observed in Fig. 2(a) that in lightly loaded scenario EDF/FCFS with feasibility admission control and aggressive reserve dispatch perform reasonably good compared with TAGS. However, in overloaded scenario the performance of EDF/FCFS with aggressive reserve dispatch degrade significantly, due to excessive usage of the expensive reserve energy $\left(c=1.9, v(r, d, p)=p\left(1+1_{\{(d-r) / p<2\}}\right)\right)$. Fig. 2(b) depicts the performance of EDF/FCFS with feasibility admission control and conservative reserve dispatch. While the performance in lightly loaded scenario is similar to that in Fig. 2(a), EDF/FCFS with conservative reserve dispatch degrade mildly under overwhelming arrival rate. The gap between TAGS and EDF/FCFS with conservative reserve dispatch in overloaded scenario can be attributed to the reluctancy to sacrifice the previously accepted requests for later releases with more profitability. Under overwhelming arrival rate there is a significant chance that an online operator will find himself in the dilemma of previously accepted, and more profitable later requests. Fig. 2(b) indicates that TAGS resolves the tradeoff with satisfactory profit performance.

\section{CONCLUSION}

We consider EV charging problem with renewable energy sources where charging requests arrive sporadically with deadlines and charging level requirement. We consider the reserve

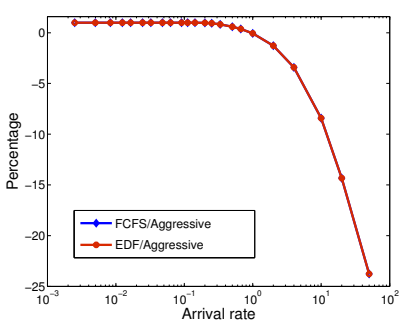

(a) EDF/FCFS, aggressive reserve (b) EDF/FCFS, conservative reserve dispatch

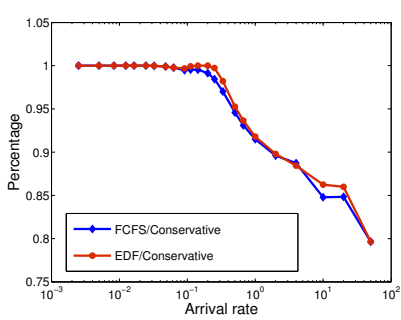

dispatch
Figure 2. Average performance

dispatch, admission and scheduling aspects of the charging facility operations, and propose a profit based reserve dispatch algorithm, together with an online admission and scheduling algorithm TAGS. Satisfactory average performance of TAGS is demonstrated for both underloaded and overloaded scenarios via comparative simulation with benchmark algorithms such as $\mathrm{EDF} / \mathrm{FCFS}$ with aggressive/conservative reserve dispatch algorithms.

\section{REFERENCES}

[1] L. Gan, U. Topcu, and S. H. Low, "Optimal decentralized protocol for electric vehicle charging," in Proceedings of the 50th IEEE Conference on Decision and Control, 2011.

[2] S. Sojoudi and S. H. Low, "Optimal charging of plug-in hybrid electric vehicles in smart grids," in Proc. IEEE PES General Meeting, 2011.

[3] W. Su and M.-Y. Chow, "Performance evaluation of a PHEV parking station using particle swarm optimization," in IEEE Power and Energy Society General Meeting 2011, Detroit, MI, USA, July 2011.

[4] A. Subramanian, M. Garcia, A. Dominguez-Garcia, D. Callaway, K. Poolla, and P. Varaiya, "Real-time scheduling deferrable electric loads," in 2012 American Control Conference, 2012.

[5] M. Caramanis and J. M. Foster, "Management of electric vehicle charging to mitigate renewable generation intermittency and distribution network congestion," in Proceedings of the 48th IEEE Conference on Decision and Control, Dec 2009, pp. 4717-4722.

[6] M. Kefayati and C. Caramanis, "Efficient energy delivery management for PHEVs," in 2010 First IEEE International Conference on Smart Grid Communications (SmartGridComm), Oct 2010, pp. 525-530.

[7] C. Pang, P. Dutta, S. Kim, M. Kezunovic, and I. Damnjanovic, "PHEVs as dynamically configurable dispersed energy storage for V2B uses in the smart grid," in 7th Mediterranean Conference and Exhibition on Power Generation, Transmission, Distribution and Energy Conversion (MedPower 2010), Nov 2010, pp. 1-6.

[8] Z. Ma, D. S. Callaway, and I. A. Hiskens, "Decentralized charging control of large populations of plug-in electric vehicles," IEEE Trans. Control Systems Technology, 2011.

[9] S. Chen, T. He, and L. Tong, "Optimal deadline scheduling with commitment," in 2011 Allerton Conference on Communication, Control and Computing, Oct 2011.

[10] S. Chen, Y. Ji, and L. Tong, "Large scale charging of electric vehicles," 2012, accepted by Proc. IEEE PES General Meeting.

[11] C. L. Liu and J. W. Layland, "Scheduling algorithms for multiprogramming in a hard-real-time environment," Journal of ACM, vol. 20, pp. 46-61, 1973.

[12] A. Mok, "Fundamental design problems of distributed systems for the hard real-time environment," Ph.D. dissertation, MIT, 1983.

[13] C. D. Locke, "Best-effort decision-making for real-time scheduling," Ph.D. dissertation, CMU, 1986.

[14] G. Koren and D. Shasha, "Dover: An optimal on-line scheduling algorithm for overloaded uniprocessor real-time systems," SIAM Journal of Computing, vol. 24, pp. 318-339, 1995. 